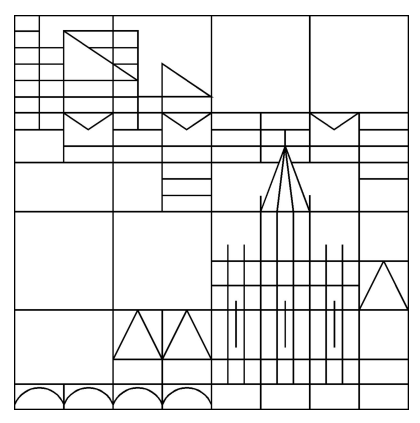

Decay estimates for the Cauchy problem for the damped extensible beam equation

\author{
Reinhard Racke \\ Shuji Yoshikawa
}

Konstanzer Schriften in Mathematik

Nr. 335, Februar 2015

ISSN 1430-3558 



\title{
Decay estimates for the Cauchy problem for the damped extensible beam equation
}

\author{
Reinhard Racke, ${ }^{*} \quad$ Shuji Yoshikawa, ${ }^{\dagger}$
}

\begin{abstract}
The extensible beam equation proposed by Woinovsky-Krieger [13] is a fourth order dispersive equation with nonlocal nonlinear terms. In this paper we study the Cauchy problem of the extended model by Ball who proposed the following model with external and structural damping terms:

$$
\rho \partial_{t}^{2} u+\delta \partial_{t} u+\kappa \partial_{x}^{4} u+\eta \partial_{t} \partial_{x}^{4} u=\left(\alpha+\beta \int_{\mathbb{R}}\left|\partial_{x} u\right|^{2} d x+\gamma \eta \int_{\mathbb{R}} \partial_{t} \partial_{x} u \partial_{x} u d x\right) \partial_{x}^{2} u
$$

For $\eta>0$ this represents a Kelvin-Voigt damping. We show the unique global existence of solution for this problem and give a precise description of the decay of solutions in time.
\end{abstract}

\section{Introduction}

The nonlinear beam equation

$$
\rho \partial_{t}^{2} u+\kappa \partial_{x}^{4} u=\left(\alpha+\beta \int_{0}^{l}\left|\partial_{x} u\right|^{2} d x\right) \partial_{x}^{2} u
$$

was proposed by Woinovsky-Krieger [13] (see also [6]) as a model for the transverse defection $u$ of an extensible beam of natural length $l$, where $\rho, \kappa, \alpha$ and $\beta$ are positive constants. In this article we study the initial value problem for the modified model of (1.1) proposed by Ball [2], where he assumes that the beam has linear structural (Kelvin-Voigt) and external (frictional) damping, that is, we consider the following problem:

$$
\left\{\begin{array}{l}
\rho \partial_{t}^{2} u+\kappa \partial_{x}^{4} u+\delta \partial_{t} u+\eta \partial_{t} \partial_{x}^{4} u \\
\quad=\left(\alpha+\beta \int_{\mathbb{R}}\left|\partial_{x} u\right|^{2} d x+\gamma \eta \int_{\mathbb{R}} \partial_{x} u \partial_{t} \partial_{x} u d x\right) \partial_{x}^{2} u, \quad(t, x) \in \mathbb{R}^{+} \times \mathbb{R}, \\
u(0, \cdot)=f, \quad \partial_{t} u(0, \cdot)=g, \quad x \in \mathbb{R},
\end{array}\right.
$$

where $\gamma$ and $\delta$ are positive and $\eta$ is a non-negative constant. According to the modeling in [2], physically, in connection with the Kelvin-Voigt damping term $\eta \partial_{t} \partial_{x}^{4} u$, the additional

*Department of Mathematics and Statistics, University of Konstanz, 78457 Konstanz, Germany, E-mail: reinhard.racke@uni-konstanz.de

${ }^{\dagger}$ Department of Engineering for Production and Environment, Graduate School of Science and Engineering, Ehime University, 3 Bunkyo-cho, Matsuyama, Ehime, 790-8577 Japan, E-mail: yoshikawa@ehime-u.ac.jp 
nonlocal term $\gamma \eta \int_{\mathbb{R}} \partial_{x} u \partial_{t} \partial_{x} u d x$ arises. Our problem approximates a sufficiently long beam the cross-sectional area of which is proportional to the length. The initial-boundary value problem for the equation (1.1) has been studied by many authors (see e.g. [5] and [1], etc.).

On the other hand, there seem to be no results studying the initial value problem $x \in \mathbb{R}$, even if $\eta=0$. For the problem (1.2) we investigate the decay property of solutions with the help of the technique from the damped wave equation with absorbing type nonlinearity.

Before stating our main results, we explain several related results. The damped wave equation $\partial_{t}^{2} u-\Delta u+\partial_{t} u=f$ has been extensively studied. When we consider the Cauchy problem $\left(x \in \mathbb{R}^{n}\right)$ of semilinear damped wave equations with absorbing type nonlinearity $f(u)=-|u|^{p-1} u$, it is important that we find suitable techniques to utilize the dissipative property of the linearized equation. Nakao [9] proposed a technique dividing the time energy integral appropriately estimating local portions. His technique has been used and further developed by many authors (see [8], [10] etc.). Combining their techniques with the decay estimate for the linearized equation, we obtain the following result.

Theorem 1.1 (Decay estimates). Let $k \geq 2$ be an any integer and set

$$
\theta_{\ell}:=\min \left\{\frac{\ell}{2}, 2\right\}, \quad \widetilde{\theta}_{\ell}:= \begin{cases}\frac{\ell}{2}, & \ell=0,1,2,3 \\ \max _{m=3,4, \ldots, \ell} \min \left\{\widetilde{\theta}_{\ell+2-m}+1, \frac{m}{2}\right\} & \ell \geq 4 .\end{cases}
$$

1. Let $\eta=0$. For any $(f, g) \in H^{k} \times H^{k-2}$, there exists unique global solution u to (1.2) satisfying $u \in C\left([0, \infty), H^{k}\right)$ and $\partial_{t} u \in C\left([0, \infty), H^{k-2}\right)$. Moreover, the solution satisfies

$$
\left\|\partial_{x}^{\ell} u(t)\right\|_{L^{2}} \leq \frac{C_{k}}{(t+1)^{\theta_{\ell}}} \quad(0 \leq \ell \leq k), \quad\left\|\partial_{t} \partial_{x}^{m} u(t)\right\|_{L^{2}} \leq \frac{C_{k}}{(t+1)^{\theta_{m+2}}} \quad(0 \leq m \leq k-2),
$$

where $C_{k}=C\left(\|f\|_{H^{k}},\|g\|_{H^{k-2}}\right)$.

2. Let $\eta>0$. For any $(f, g) \in H^{k} \times H^{k}$ there exists unique global solution u to (1.2) satisfying $u \in C\left([0, \infty), H^{k}\right)$ and $\partial_{t} u \in C\left([0, \infty), H^{k}\right)$. Moreover, the solution satisfies

$$
\left\|\partial_{x}^{\ell} u(t)\right\|_{L^{2}} \leq \frac{\widetilde{C}_{k}}{(t+1)^{\widetilde{\theta}_{\ell}}}, \quad\left\|\partial_{t} \partial_{x}^{\ell} u(t)\right\|_{L^{2}} \leq \frac{\widetilde{C}_{k}}{(t+1)^{\widetilde{\theta}_{\ell+2}}} \quad(0 \leq \ell \leq k),
$$

where $\widetilde{C}_{k}=C\left(\|f\|_{H^{k}},\|g\|_{H^{k}}\right)$.

In the case $\eta=0$, Brito [4] considered the abstract form

$$
\rho \partial_{t}^{2} u+\kappa A^{2} u+\delta \partial_{t} u-\left(\alpha+M\left(\left|A^{\frac{1}{2}} u\right|^{2}\right)\right) A u,
$$

and showed the exponential decay of solutions if $\delta^{2} \neq 2 \alpha \zeta$ where he assumed that, with $\zeta>0,(A u, u) \geq \zeta|u|^{2}$. Biler [3] gave a remark related to improvements of the assumption in [4]. In the above results the boundedness of the domain plays an essential role. In our setting $x \in \mathbb{R}$, however, we can not expect the exponential decay of solutions. Indeed, the result [12] showed that the decay of solution is polynomially and it is optimal even in the linear case. In addition, from the result [12] we guess that the decay for $\left\|\partial_{x} u(t)\right\|_{L^{2}}$ in 
Theorem 1.1 is optimal unless we do not assume some other restriction on the data. To obtain the decay estimates in Theorem 1.1, the lower order term plays an essential role. Indeed, if we assumed $\alpha=0$, we only expect to be able to show the slower decay estimate $\left\|\partial_{x} u(t)\right\|_{L^{2}} \leq C(t+1)^{-1 / 4}$ as mentioned in [12]. In the case $\eta>0$, although Ball [2] gave an existence result in the bounded domain case, there seems to be not any results for the Cauchy problem $(x \in \mathbb{R})$ even for the linearized case. The Kelvin-Voigt damping changes the structure of the equation (1.2). For example, when we compare the linear estimates (2.9) and (2.13), the later estimate for the case $\eta>0$ can be observed a kind of regularity gain property. The intricate exponent $\widetilde{\theta}_{\ell}$ is concretely given as $\widetilde{\theta}_{\ell}=2(\ell=4,5), 5 / 2(\ell=6,7), 3$ $(\ell=8,9,10), 7 / 2(\ell=11,12,13), 4(\ell=14), \ldots$ As we will show in the proof of Lemma 3.3, it satisfies $\widetilde{\theta}_{\ell-1} \leq \widetilde{\theta}_{\ell} \leq \widetilde{\theta}_{\ell-2}+1$.

This paper is organized as follows. In Section 2 we introduce several lemmas needed later on to show the main results. In Section 3 we present the proof of the main result.

We conclude the introduction by giving notation used in this paper. We use the notation $\partial_{t}:=\frac{\partial}{\partial t}$ and $\partial_{x}:=\frac{\partial}{\partial x}$. We denote several positive constants by $C$ and $C_{i}(i=1,2,3, \ldots)$. The constant may change from line to line. Important dependencies of constants are denoted by $C=C(\ldots) . L^{p}$ and $H^{s}$ are the standard Lebesgue and Sobolev spaces, respectively. We denote the Fourier and the Fourier inverse transforms by $\mathcal{F}$ and $\mathcal{F}^{-1}$ and the Fourier transform of a function $f$ by $\widehat{f}$.

\section{Preliminaries}

In this section we introduce some useful lemmas and linear estimates. To derive the decay estimate for nonlinear problem, we use the following modified Nakao inequality introduced by Ono [10].

Lemma 2.1 ([10, Lemma 2.1]). Let $\phi$ be a nonnegative function on $[0, \infty)$, satisfying

$$
\sup _{s \in[t, t+1]} \phi(s)^{2} \leq\left\{k_{0} \phi(t)+k_{1}(t+1)^{-a}\right\}\{\phi(t)-\phi(t+1)\}+k_{2}(t+1)^{-b}
$$

for some $k_{0}, k_{1}, k_{2}, a$ and $b \geq 0$. Then $\phi$ has the decay property

$$
\phi(t) \leq C(t+1)^{-\theta}, \quad \theta=\min \{a+1, b / 2\},
$$

where $C$ denotes a positive constant depending on $\phi(0)$ and the known constants $a, b, \ldots$ appearing. In the case $k_{2}=0,(2.1)$ holds for $\theta=a+1$.

The following inequalities are well-known and useful for the estimate of the nonlinear terms.

Lemma 2.2 (see e.g. [11, Lemma 2.4]). 1. Let $a>0$ and $b>0$ with $\min \{a, b\}>1$. It holds

$$
\int_{0}^{t}(t-s+1)^{-a}(s+1)^{-b} d s \leq C(t+1)^{-\min \{a, b\}} .
$$

2. Let $1>a \geq 0, b>0$ and $c>0$. It holds

$$
\int_{0}^{t} e^{-c(t-s)}(t-s)^{-a}(s+1)^{-b} d s \leq C(t+1)^{-b} .
$$


For short, throughout this paper we often denote

$$
I(u):=\int_{\mathbb{R}}\left|\partial_{x} u\right|^{2} d x, \quad \widetilde{I}(u):=\int_{\mathbb{R}} \partial_{x} u \partial_{t} \partial_{x} u d x .
$$

We define the mild solution to (1.2) in the case $\rho>0$ by the solution of the following integral equation in the $L^{2}$-sense

$$
u(t)=\mathcal{K}_{0}(t) f+\mathcal{K}_{1}(t) g+\frac{1}{\rho} \int_{0}^{t} \mathcal{K}_{1}(t-s)\{\beta I(u)+\gamma \eta \widetilde{I}(u)\} \partial_{x}^{2} u(s) d s,
$$

where $\mathcal{K}_{0}(t) f:=\mathcal{F}^{-1}\left[K_{0}(t, \xi) \widehat{f}\right]$ and $\mathcal{K}_{1}(t) f:=\mathcal{F}^{-1}\left[K_{1}(t, \xi) \widehat{f}\right]$ with

$$
\begin{aligned}
& K_{0}(t, \xi):= e^{-\frac{1}{2} a(\xi) t} \frac{e^{\frac{\sqrt{a(\xi)^{2}-4 b(\xi)}}{2} t}+e^{-\frac{\sqrt{a(\xi)^{2}-4 b(\xi)}}{2} t}}{2} \\
&+\frac{a(\xi) e^{-\frac{1}{2} a(\xi) t}}{2 \sqrt{a(\xi)^{2}-4 b(\xi)}}\left(e^{\frac{\sqrt{a(\xi)^{2}-4 b(\xi)}}{2} t}-e^{-\frac{\sqrt{a(\xi)^{2}-4 b(\xi)}}{2} t}\right), \\
& K_{1}(t, \xi):= \frac{e^{-\frac{1}{2} a(\xi) t}}{\sqrt{a(\xi)^{2}-4 b(\xi)}}\left(e^{\frac{\sqrt{a(\xi)^{2}-4 b(\xi)}}{2} t}-e^{-\frac{\sqrt{a(\xi)^{2}-4 b(\xi)}}{2} t}\right), \\
& a(\xi):=\frac{\delta}{\rho}+\frac{\eta}{\rho} \xi^{4}, \quad b(\xi):=\frac{\kappa}{\rho} \xi^{4}+\frac{\alpha}{\rho} \xi^{2} .
\end{aligned}
$$

To prove a local existence theorem and decay estimates, we use $L^{2}-L^{2}$ estimate which can be proved by the standard Fourier splitting method. Although we could derive $L^{p}-L^{q}$ estimate by using the Carlson-Beurling inequality as in [7] and [11], we only state $L^{2}-L^{2}$ case for simplicity because we use only this case in this article.

Proposition 2.3. Let $k$ be an any nonnegative integer and $\mathcal{K}_{0}$ and $\mathcal{K}_{1}$ be defined by (2.3) and (2.4).

1. If $\eta=0$, then it holds that for $0 \leq \ell \leq k$ and $0 \leq n \leq \min \{k, 2\}$

$$
\begin{aligned}
\left\|\partial_{x}^{k} \mathcal{K}_{0}(t) f\right\|_{L^{2}} & \leq \frac{C}{(t+1)^{\frac{\ell}{2}}}\left\|\partial_{x}^{k-\ell} f\right\|_{L^{2}}+C e^{-C t}\left\|\partial_{x}^{k} f\right\|_{L^{2}}, \\
\left\|\partial_{x}^{k} \mathcal{K}_{1}(t) f\right\|_{L^{2}} & \leq \frac{C}{(t+1)^{\frac{\ell}{2}}}\left\|\partial_{x}^{k-\ell} f\right\|_{L^{2}}+C e^{-C t}\left\|\partial_{x}^{k-n} f\right\|_{L^{2}} . \\
\left\|\partial_{x}^{k} \partial_{t} \mathcal{K}_{0}(t) f\right\|_{L^{2}} & \leq \frac{C}{(t+1)^{\frac{\ell}{2}+1}}\left\|\partial_{x}^{k-\ell} f\right\|_{L^{2}}+C e^{-C t}\left\|\partial_{x}^{k} f\right\|_{L^{2}}, \\
\left\|\partial_{x}^{k} \partial_{t} \mathcal{K}_{1}(t) f\right\|_{L^{2}} & \leq \frac{C}{(t+1)^{\frac{\ell}{2}+1}}\left\|\partial_{x}^{k-\ell} f\right\|_{L^{2}}+C e^{-C t}\left\|\partial_{x}^{k} f\right\|_{L^{2}} .
\end{aligned}
$$


2. If $\eta>0$, then it holds that for $0 \leq \ell, m \leq k$ and $0 \leq n \leq \min \{k, 4\}$

$$
\begin{gathered}
\left\|\partial_{x}^{k} \mathcal{K}_{0}(t) f\right\|_{L^{2}} \leq \frac{C}{(t+1)^{\frac{\ell}{2}}}\left\|\partial_{x}^{k-\ell} f\right\|_{L^{2}}+C e^{-C t}\left\|\partial_{x}^{k} f\right\|_{L^{2}}, \\
\left\|\partial_{x}^{k} \mathcal{K}_{1}(t) f\right\|_{L^{2}} \leq \frac{C}{(t+1)^{\frac{\ell}{2}}}\left\|\partial_{x}^{k-\ell} f\right\|_{L^{2}}+C e^{-C t}\left\|\partial_{x}^{k-n} f\right\|_{L^{2}}, \\
\left\|\partial_{x}^{k} \partial_{t} \mathcal{K}_{0}(t) f\right\|_{L^{2}} \leq \frac{C}{(t+1)^{\frac{\ell}{2}+1}}\left\|\partial_{x}^{k-\ell} f\right\|_{L^{2}}+C e^{-C t}\left\|\partial_{x}^{k} f\right\|_{L^{2}}, \\
\left\|\partial_{x}^{k} \partial_{t} \mathcal{K}_{1}(t) f\right\|_{L^{2}} \leq \frac{C}{(t+1)^{\frac{\ell}{2}+1}}\left\|\partial_{x}^{k-\ell} f\right\|_{L^{2}}+\frac{C}{e^{C t}}\left\|\partial_{x}^{k-n} f\right\|_{L^{2}}+\frac{C}{t^{m / 4} e^{C t}}\left\|\partial_{x}^{k-m} f\right\|_{L^{2}}
\end{gathered}
$$

Proof. We first prove the inequalities (2.10)-(2.13) for the case $\eta>0$. Since from (2.5)

$$
a(\xi)^{2}-4 b(\xi)=\frac{\eta^{2}}{\rho^{2}} \xi^{8}+\left(\frac{2 \delta \eta}{\rho^{2}}-\frac{4 \kappa}{\rho}\right) \xi^{4}-\frac{4 \alpha}{\rho} \xi^{2}+\frac{\delta^{2}}{\rho^{2}}
$$

we may choose $r \leq 1$ small satisfying $a(\xi)^{2}-4 b(\xi) \geq \exists c_{r}>0$ for all $|\xi| \leq r$, and large $R>\max \left\{\sqrt{\frac{\delta \kappa}{\alpha \eta}}, 1\right\}$ satisfying $a^{2}(\xi)-4 b(\xi) \geq \exists c_{R}|\xi|^{8}>0$ for all $|\xi| \geq R$.

We start proving the inequality (2.11). Observe that $\left.|| \xi\right|^{k} e^{-C|\xi|^{a} t} \mid \leq C / t^{k / a}$ and that for $a^{2}-4 b>0$

$$
\frac{a(\xi)}{2}-\frac{\sqrt{a^{2}(\xi)-4 b(\xi)}}{2}=\frac{2 b(\xi)}{a(\xi)+\sqrt{a^{2}(\xi)-4 b(\xi)}} \geq \frac{b(\xi)}{a(\xi)}
$$

Since $\frac{b(\xi)}{a(\xi)}=\frac{\kappa \xi^{4}+\alpha \xi^{2}}{\delta+\eta \xi^{4}} \geq \frac{\alpha}{\delta+\eta r^{4}} \xi^{2}(|\xi| \leq r)$, we have

$$
\begin{aligned}
\left.|| \xi\right|^{k} K_{1}(t, \xi) \mid & \leq\left|\frac{|\xi|^{k}}{\sqrt{a^{2}(\xi)-4 b(\xi)}}\left\{e^{-\left(\frac{a(\xi)}{2}-\frac{\sqrt{a^{2}(\xi)-4 b(\xi)}}{2}\right) t}-e^{-\left(\frac{a(\xi)}{2}+\frac{\sqrt{a^{2}(\xi)-4 b(\xi)}}{2}\right) t}\right\}\right| \\
& \leq e^{\frac{\alpha}{\delta+\eta r^{4}} r^{2}}\left|\frac{|\xi|^{k}}{\sqrt{c_{r}}} e^{-\frac{\alpha}{\delta+\eta r^{4}} \xi^{2}(t+1)}\right|+\frac{|r|^{k}}{\sqrt{c_{r}}} e^{-\frac{\delta}{2 \rho} t} \leq \frac{C}{(t+1)^{\frac{k}{2}}} \quad(|\xi| \leq r) .
\end{aligned}
$$

We see that for $|\xi| \geq R$

$$
\frac{a(\xi)}{2}-\frac{\sqrt{a^{2}(\xi)-4 b(\xi)}}{2} \geq \frac{\kappa}{\eta}
$$

since $\eta b(\xi) \geq \kappa a(\xi)$ holds by the assumption on $R$. Then for $|\xi| \geq R$ we have

$$
e^{-\left(\frac{a(\xi)}{2}-\frac{\sqrt{a^{2}(\xi)-4 b(\xi)}}{2}\right) t} \leq e^{-\frac{\kappa}{\eta} t}, \quad e^{-\left(\frac{a(\xi)}{2}+\frac{\sqrt{a^{2}(\xi)-4 b(\xi)}}{2}\right) t} \leq e^{-\frac{\delta}{2 \rho} t} .
$$

Therefore we obtain

$$
\left.\left.|| \xi\right|^{k} K_{1}(t, \xi)\left|\leq \frac{|\xi|^{k}}{\sqrt{c_{R}}|\xi|^{4}}\left(e^{-\frac{\kappa}{\eta} t}+e^{-\frac{\delta}{2 \rho} t}\right) \leq C\right| \xi\right|^{k-4} e^{-C t}
$$

Since the function $\frac{\sinh x}{x}$ is bounded on bounded sets, we see that for $r \leq|\xi| \leq R$

$$
\left.\left.|| \xi\right|^{k} K_{1}(t, \xi)\left|\leq \frac{t|\xi|^{k}}{2} e^{-\frac{a(\xi)}{2} t}\right| \frac{e^{\frac{\sqrt{a^{2}(\xi)-4 b(\xi)}}{2}}-e^{-\frac{\sqrt{a^{2}(\xi)-4 b(\xi)}}{2}} t}{\frac{\sqrt{a^{2}(\xi)-4 b(\xi)}}{2} t}|\leq C| R\right|^{k} t e^{-\frac{\delta}{2 \rho} t} \leq C e^{-C t}
$$


Consequently it follows from Plancherel's theorem that

$$
\begin{aligned}
\left\|\partial_{x}^{k} \mathcal{K}_{1}(t) f\right\|_{L^{2}} \leq & \left.\sup _{|\xi| \leq r}|| \xi\right|^{k} K_{1}(t, \xi)\left|\|f\|_{L^{2}}+\sup _{r \leq|\xi| \leq R}\right||\xi|^{k} K_{1}(t, \xi) \mid\|f\|_{L^{2}} \\
& +\left.\sup _{R \leq|\xi|}|| \xi\right|^{4} K_{1}(t, \xi) \mid\left\|\partial_{x}^{k-4} f\right\|_{L^{2}} \leq \frac{C}{(t+1)^{\frac{k}{2}}}\|f\|_{L^{2}}+C e^{-C t}\left\|\partial_{x}^{k-4} f\right\|_{L^{2}},
\end{aligned}
$$

due to (2.15), (2.17) and (2.18). This proves (2.11).

Next we show (2.10). From the same argument as (2.15) we have for $|\xi| \leq r$

$$
\left.|| \xi\right|^{k} K_{0}(t, \xi) \mid \leq C\left(|\xi|^{k}+|\xi|^{k+2}+|\xi|^{k+4}\right) e^{-\frac{\alpha}{\delta+\eta r^{4}} \xi^{2}(t+1)}+C e^{-\frac{\delta}{2 \rho} t} \leq \frac{C}{(t+1)^{\frac{k}{2}}} .
$$

Since $\frac{a(\xi)}{\sqrt{a^{2}(\xi)-4 b(\xi)}} \leq \frac{\delta+\eta|\xi|^{4}}{\rho \sqrt{c_{R}|\xi|^{4}}}(|\xi| \geq R)$, we have

$$
\left.\left.|| \xi\right|^{k} K_{1}(t, \xi)\left|\leq C\left(|\xi|^{k-4}+|\xi|^{k}\right)\left(e^{-\frac{\kappa}{\eta} t}+e^{-\frac{\delta}{2 \rho} t}\right) \leq C\right| \xi\right|^{k} e^{-C t} \quad(|\xi|>R),
$$

in a similar manner to (2.17). It holds that for $r \leq|\xi| \leq R$

$$
\begin{aligned}
\left.|| \xi\right|^{k} K_{0}(t, \xi) \mid & \leq \frac{R^{k}}{e^{\frac{\delta}{2 \rho} t}}\left|\frac{e^{\frac{\sqrt{a(\xi)^{2}-4 b(\xi)}}{2}}+e^{-\frac{\sqrt{a(\xi)^{2}-4 b(\xi)}}{2} t}}{2}\right|+\frac{t R^{k} a(R)}{4 e^{\frac{\delta}{2 \rho} t}}\left|\frac{e^{\frac{\sqrt{a^{2}(\xi)-4 b(\xi)}}{2} t}-e^{-\frac{\sqrt{a^{2}(\xi)-4 b(\xi)}}{2} t}}{\frac{\sqrt{a^{2}(\xi)-4 b(\xi)}}{2} t}\right| \\
& \leq C e^{-C t}+C t e^{-C t} \leq C e^{-C t} .
\end{aligned}
$$

Then (2.10) follows from (2.19), (2.20) and (2.21).

Next, we show the estimate (2.12). Observe that

$$
\partial_{t} K_{0}(t, \xi)=-\frac{b(\xi)}{\sqrt{a(\xi)^{2}-4 b(\xi)}} e^{-\frac{a(\xi)}{2} t}\left(e^{\frac{\sqrt{a(\xi)^{2}-4 b(\xi)}}{2} t}-e^{-\frac{\sqrt{a(\xi)^{2}-4 b(\xi)}}{2} t}\right) .
$$

We have $\left.\left.|| \xi\right|^{k} \partial_{t} K_{0}(t, \xi)\left|\leq \frac{\kappa+\alpha}{\rho \sqrt{c_{r}}}\right| \xi\right|^{k+2} e^{-\frac{\alpha}{\delta+\eta r^{4}} \xi^{2} t}+\frac{b(r) r^{k}}{\sqrt{c_{r}}} e^{-\frac{\delta}{2 \rho} t} \leq \frac{C}{(t+1)^{\frac{k}{2}+1}}(|\xi| \leq r \leq 1)$, and $\left.\left.|| \xi\right|^{k} \partial_{t} K_{0}(t, \xi)\left|\leq\left(\frac{\alpha|\xi|^{k+2}+\left.\kappa|\xi|\right|^{k+4}}{2 \rho \sqrt{c_{C} \xi^{4}}}\right)\left(e^{-\frac{\kappa}{\eta} t}+e^{-\frac{\delta}{2 \rho} t}\right) \leq C\right| \xi\right|^{k} e^{-C t}(|\xi| \geq R)$. From the same argument as in the estimates for $K_{1}((2.18))$, it immediately follows that $\left.|| \xi\right|^{k} \partial_{t} K_{0}(t, \xi) \mid \leq$ $C t e^{-C t} \leq C e^{-C t}$ for $r \leq|\xi| \leq R$. Then we arrive at (2.12).

Next we show (2.13). Observe that

$$
\begin{aligned}
& \partial_{t} K_{1}(t, \xi) \\
& =\left\{\frac{e^{\frac{\sqrt{a(\xi)^{2}-4 b(\xi)}}{2}}+e^{-\frac{\sqrt{a(\xi)^{2}-4 b(\xi)}}{2}} t}{2}-\frac{a(\xi)}{2 \sqrt{a(\xi)^{2}-4 b(\xi)}}\left(e^{\frac{\sqrt{a(\xi)^{2}-4 b(\xi)}}{2} t}-e^{-\frac{\sqrt{a(\xi)^{2}-4 b(\xi)}}{2} t}\right)\right\} e^{-\frac{1}{2} a(\xi) t} \\
& =\frac{-2 b(\xi)}{\sqrt{a(\xi)^{2}-4 b(\xi)}\left(\sqrt{a(\xi)^{2}-4 b(\xi)}+a(\xi)\right)} e^{-\left(\frac{a(\xi)}{2}-\frac{\sqrt{a^{2}(\xi)-4 b(\xi)}}{2}\right) t} \\
& +\frac{\sqrt{a(\xi)^{2}-4 b(\xi)}+a(\xi)}{2 \sqrt{a(\xi)^{2}-4 b(\xi)}} e^{-\left(\frac{a(\xi)}{2}+\frac{\sqrt{a^{2}(\xi)-4 b(\xi)}}{2}\right) t} .
\end{aligned}
$$


We have for $|\xi| \leq r \leq 1$

$$
\left.\left.|| \xi\right|^{k} \partial_{t} K_{1}(t, \xi)\left|\leq \frac{\kappa+\alpha}{\rho c_{r}}\right| \xi\right|^{k+2} e^{-\frac{\alpha}{\delta+\eta r^{4}} \xi^{4} t}+\frac{a(r) r^{k}}{\sqrt{c_{r}}} e^{-\frac{\delta}{2 \rho} t} \leq \frac{C}{(t+1)^{\frac{k}{2}+1}},
$$

because

$$
\begin{gathered}
\left|\frac{2 b(\xi)}{\sqrt{a(\xi)^{2}-4 b(\xi)}\left(\sqrt{a(\xi)^{2}-4 b(\xi)}+a(\xi)\right)}\right| \leq \frac{b(\xi)}{a(\xi)^{2}-4 b(\xi)} \leq \frac{\kappa+\alpha}{\rho c_{r}} \xi^{2}, \\
\left|\frac{\sqrt{a(\xi)^{2}-4 b(\xi)}+a(\xi)}{2 \sqrt{a(\xi)^{2}-4 b(\xi)}}\right| \leq \frac{a(\xi)}{\sqrt{a(\xi)^{2}-4 b(\xi)}} \leq \frac{a(r)}{\sqrt{c_{r}}} .
\end{gathered}
$$

For $|\xi| \geq R \geq 1$ we obtain from $(2.16)$

$$
\begin{aligned}
& \left|\frac{2 b(\xi)}{\sqrt{a(\xi)^{2}-4 b(\xi)}\left(\sqrt{a(\xi)^{2}-4 b(\xi)}+a(\xi)\right)} e^{-\left(\frac{a(\xi)}{2}-\frac{\sqrt{a^{2}(\xi)-4 b(\xi)}}{2}\right) t}\right| \\
& \quad \leq \frac{b(\xi)}{a(\xi)^{2}-4 b(\xi)} e^{-\frac{\kappa}{\eta} t} \leq \frac{\kappa+\alpha}{\rho c_{R}} e^{-\frac{\kappa}{\eta} t} \xi^{-4}
\end{aligned}
$$

and

$$
\begin{gathered}
\left|\frac{a(\xi)+\sqrt{a^{2}(\xi)-4 b(\xi)}}{2 \sqrt{a^{2}(\xi)-4 b(\xi)}} e^{-\left(\frac{a(\xi)}{2}+\frac{\sqrt{a^{2}(\xi)-4 b(\xi)}}{2}\right) t}\right| \leq \frac{a(\xi)}{\sqrt{a^{2}(\xi)-4 b(\xi)}} e^{-\frac{a(\xi)}{2} t} \\
\quad \leq \frac{\delta+\eta}{\rho \sqrt{c_{R}}} e^{-\frac{\delta}{2 \rho} t} e^{-\frac{\eta}{2 \rho} \xi^{4} t} \leq\left.\left. C e^{-C t}|\xi|^{-m}|| \xi\right|^{m} e^{-C t \xi^{4}}\left|\leq \frac{C}{t^{m / 4} e^{C t}}\right| \xi\right|^{-m} .
\end{gathered}
$$

Then we obtain for nonnegative integers $k$ and $\ell$ and an integer $n \in[0,4]$

$$
\begin{aligned}
& \left.|| \xi\right|^{k} \partial_{t} K_{1}(t, \xi)|\leq| \frac{|\xi|^{k-4}}{\sqrt{c_{R}}}\left(\frac{a(\xi)}{2}-\frac{\sqrt{a^{2}(\xi)-4 b(\xi)}}{2}\right) e^{-\left(\frac{a(\xi)}{2}-\frac{\sqrt{a^{2}(\xi)-4 b(\xi)}}{2}\right) t} \mid \\
& \quad+\left|\frac{|\xi|^{k-4}}{\sqrt{c_{R}}}\left(\frac{a(\xi)}{2}+\frac{\sqrt{a^{2}(\xi)-4 b(\xi)}}{2}\right) e^{-\left(\frac{a(\xi)}{2}+\frac{\sqrt{a^{2}(\xi)-4 b(\xi)}}{2}\right) t}\right| \leq \frac{C}{e^{C t}}|\xi|^{k-n}+\frac{C}{t^{m / 4} e^{C t}}|\xi|^{k-m} .
\end{aligned}
$$

From the same argument as in (2.21) we see that $\left.|| \xi\right|^{k} \partial_{t} K_{1}(t, \xi) \mid \leq C t e^{-C t}+C e^{-C t} \leq C e^{-C t}$ $(r \leq|\xi| \leq R)$. Then we can establish (2.13).

In the last part of the proof, we consider now the case $\eta=0$. The inequalities (2.6) and (2.7) can be found in Propositions 3.1 and 3.2 in [11] as a special case $(q=r=2)$. Therefore we only show (2.8) and (2.9). Let us choose small $r<1$ satisfying $a^{2}-4 b(r)>0$ and $R>1$ satisfying $a^{2}-4 b(R)<0$. We write $\widetilde{c}_{r}:=a^{2}-4 b(r)$ and define $\widetilde{c}_{R}$ by a positive constant satisfying $a^{2}-4 b(\xi)<-\widetilde{c}_{R} \xi^{4}$ for all $|\xi|>R$. Recalling (2.22), we have for $|\xi|<r<1$

$$
\left.|| \xi\right|^{k} \partial_{t} K_{0}(t, \xi) \mid \leq \frac{2(\kappa+\alpha)|\xi|^{k+2}}{\sqrt{a^{2}-4 b(r)}} e^{-\frac{\alpha}{\delta} \xi^{2} t}+\frac{b(r)}{\sqrt{a^{2}-4 b(r)}} e^{-\frac{\delta}{2 \rho} t} \leq \frac{C}{(t+1)^{\frac{k}{2}+1}} .
$$


It holds that for $|\xi|>R>1$

$$
\left.\left.|| \xi\right|^{k} \partial_{t} K_{0}(t, \xi)\left|\leq \frac{2(\kappa+\alpha)|\xi|^{k+4}}{\sqrt{\widetilde{c}_{R}} \xi^{2}} e^{-\frac{\delta}{2 \rho} t}\left(\left|e^{\frac{\sqrt{4 b(\xi)-a^{2}}}{2} i t}\right|+\left|e^{\frac{\sqrt{4 b(\xi)-a^{2}}}{2} i t}\right|\right) \leq C\right| \xi\right|^{k+2} e^{-C t} .
$$

In an argument similar to (2.18) for $r \leq|\xi| \leq R$ we see

$$
\left.|| \xi\right|^{k} \partial_{t} K_{0}(t, \xi)\left|\leq \frac{t R^{k} b(R)}{2} e^{-\frac{\delta}{2 \rho} t}\right| \frac{e^{\frac{\sqrt{a^{2}-4 b(\xi)}}{2} t}-e^{-\frac{\sqrt{a^{2}-4 b(\xi)}}{2}} t}{\frac{\sqrt{a^{2}-4 b(\xi)}}{2} t} \mid \leq C e^{-C t},
$$

with the help of the boundedness of the functions $\frac{\sinh x}{x}$ and $\frac{\sin x}{x}$ in bounded sets.

Lastly we show (2.9). Recalling (2.23) and letting $\eta=0$ in (2.24), we have for $|\xi| \leq r$

$$
\left.\left.|| \xi\right|^{k} \partial_{t} K_{1}(t, \xi)\left|\leq \frac{2(\kappa+\alpha)}{\rho\left(a^{2}-4 b(r)\right)}\right| \xi\right|^{k+2} e^{-\frac{\alpha}{\delta} \xi^{2} t}+\frac{a(r) r^{k}}{\sqrt{c_{r}}} e^{-\frac{\delta}{2 \rho} t} \leq \frac{C}{(t+1)^{\frac{k}{2}+1}},
$$

and for $|\xi| \geq R$

$$
\left.\left.|| \xi\right|^{k} \partial_{t} K_{1}(t, \xi)|\leq C| \xi\right|^{k} e^{-\frac{\delta}{2 \rho} t} .
$$

From the same argument as above, we see $\left.|| \xi\right|^{k} \partial_{t} K_{1}(t, \xi) \mid \leq C e^{-C t}(r \leq|\xi| \leq R)$. Then we obtain (2.9). This completes the proof of Proposition 2.3.

An existence result in a suitable setting can be found in Brito [4] for the case $\eta=0$ and in Ball [2] for the case $\eta>0$, for bounded domains. Although the proof for the unbounded domain case is also not too difficult, we show it here in both cases $\eta=0$ and $\eta>0$, respectively, for self-containedness. By using the local existence and the decay estimate for nonlinear problem given later, the unique global existence can then easily be shown.

Proposition 2.4 (Local existence and uniqueness). Let $k$ be an any nonnegative integer.

1. Let $\eta \geq 0$. For any $(f, g) \in H^{k+2} \times H^{k}$, there exists $T=T\left(\|f\|_{H^{k+2}},\|g\|_{H^{k}}\right)$ such that there exists a unique mild solution $u$ to (1.2) satisfying $u \in C\left([0, T], H^{k+2}\right), \partial_{t} u \in$ $C\left([0, T], H^{k}\right)$.

2. Let $\eta>0$. For any $(f, g) \in H^{k+2} \times H^{k+2}$, there exists $T=T\left(\|f\|_{H^{k+2}},\|g\|_{H^{k+2}}\right)$ such that there exists a unique mild solution $u$ to $(1.2)$ satisfying $u \in C\left([0, T], H^{k+2}\right)$, $\partial_{t} u \in C\left([0, T], H^{k+2}\right)$.

We remark that we regard $\widetilde{I}(u)$ as $-\int_{\mathbb{R}} \partial_{t} u \partial_{x}^{2} u d x$ for the problem with $\eta>0$ in $k=0$.

Proof. 1. To establish the local existence result, we define a nonlinear mapping by:

$$
\Phi[u]:=\mathcal{K}_{0}(t) f+\mathcal{K}_{1}(t) g+\frac{1}{\rho} \int_{0}^{t} \mathcal{K}_{1}(t-s)\{\beta I(u(s))+\gamma \eta \widetilde{I}(u(s))\} \partial_{x}^{2} u(s) d s
$$


and the ball $X_{T}:=\left\{u \mid\|u\|_{X} \leq M\right\}$, where $\|u\|_{X}:=\|u\|_{L_{T}^{\infty} H^{k+2}}+\left\|\partial_{t} u\right\|_{L_{T}^{\infty} H^{k}}$. We shall show that the map $\Phi$ is a contraction mapping on $X_{T}$. From Proposition 2.3 it obviously holds that

$$
\begin{gathered}
\left\|\mathcal{K}_{0}(t) f\right\|_{H^{k+2}}+\left\|\mathcal{K}_{1}(t) g\right\|_{H^{k+2}} \leq C\left(\|f\|_{H^{k+2}}+\|g\|_{H^{k}}\right), \\
\left\|\partial_{t} \mathcal{K}_{0} f\right\|_{H^{k}}+\left\|\partial_{t} \mathcal{K}_{1}(t) g\right\|_{H^{k}} \leq C\left(\|f\|_{H^{k}}+\|g\|_{H^{k}}\right), \\
\left\|\int_{0}^{t} \mathcal{K}_{1}(t-s)\{\beta I(u(s))+\gamma \eta \widetilde{I}(u(s))\} \partial_{x}^{2} u(s) d s\right\|_{H^{k+2}} \\
\quad \leq C \int_{0}^{t}\left(\left\|\partial_{x} u(s)\right\|_{L^{2}}^{2}+\left\|\partial_{t} u(s)\right\|_{L^{2}}\left\|\partial_{x}^{2} u(s)\right\|_{L^{2}}\right)\|u(s)\|_{H^{k+2}} d s, \\
\left\|\partial_{t} \int_{0}^{t} \mathcal{K}_{1}(t-s)\{\beta I(u(s))+\gamma \eta \widetilde{I}(u(s))\} \partial_{x}^{2} u(s) d s\right\|_{H^{k}} \\
\leq C \int_{0}^{t}\left(\left\|\partial_{x} u(s)\right\|_{L^{2}}^{2}+\left\|\partial_{t} u(s)\right\|_{L^{2}}\left\|\partial_{x}^{2} u(s)\right\|_{L^{2}}\right)\|u(s)\|_{H^{k+2}} d s .
\end{gathered}
$$

where in the last inequality we have used the fact $\mathcal{K}_{1}(0)=O$. Then we have

$$
\|\Phi[u]\|_{X} \leq C\left(\|f\|_{H^{k+2}}+\|g\|_{H^{k}}\right)+C T\|u\|_{X}^{3} .
$$

From the same argument we easily deduce that

$$
\begin{aligned}
\|\Phi[u]-\Phi[\widetilde{u}]\|_{X} & \leq C \sup _{0 \leq t \leq T} \int_{0}^{t}\left\|(\beta I(u)+\gamma \eta \widetilde{I}(u)) \partial_{x}^{2} u-(\beta I(\widetilde{u})+\gamma \eta \widetilde{I}(\widetilde{u})) \partial_{x}^{2} \widetilde{u}\right\|_{L^{2}} d s \\
& \leq C T\left(\|u\|_{X}^{2}+\|\widetilde{u}\|_{X}^{2}\right)\|u-\widetilde{u}\|_{X} .
\end{aligned}
$$

This implies the desired result by choosing $M=2 C\left(\|g\|_{H^{k}}+\|f\|_{H^{k+2}}\right)$ and $T<1 /\left(2 C M^{2}\right)$. 2. The same argument as above with the help of the estimate (2.13) yields the result for $\eta>0$. Indeed, if we set $\|u\|_{X}:=\|u\|_{L_{T}^{\infty} H^{k+2}}+\left\|\partial_{t} u\right\|_{L_{T}^{\infty} H^{k+2}}(k \geq 0)$, then by (2.13) as $n=m=2$ we have

$$
\begin{aligned}
& \left\|\partial_{x}^{k+2} \partial_{t} \int_{0}^{t} \mathcal{K}_{1}(t-s)\{\beta I(u(s))+\gamma \eta \widetilde{I}(u(s))\} \partial_{x}^{2} u(s) d s\right\|_{L^{2}} \\
& \quad \leq C \int_{0}^{t}\left(1+\frac{1}{(t-s)^{1 / 2} e^{C(t-s)}}\right)\left(\left\|\partial_{x} u(s)\right\|_{L^{2}}^{2}+\left\|\partial_{t} u(s)\right\|_{L^{2}}\left\|\partial_{x}^{2} u(s)\right\|_{L^{2}}\right)\left\|\partial_{x}^{k+2} u(s)\right\|_{L^{2}} d s \\
& \quad \leq C\|u\|_{X}^{3}\left(T+T^{1 / 2}\right) .
\end{aligned}
$$

Therefore by choosing small $T$ satisfying $C M^{2}\left(T+T^{1 / 2}\right)<1 / 2$, we have desired result.

\section{Asymptotic behavior}

Unique local in time existence of mild solution has been proved in Section 2, and hence, once we show a priori estimates corresponding to the local existence results, we can extend the local solution to a global one. Therefore, we concentrate on the topics on the derivation of the decay estimates (3.10) of the solution to (1.2). 
As we have already seen in Proposition 2.3, we should split the arguments into the case $\eta=0$ and the case $\eta>0$. However, the decay of the energy $E_{\rho}(t)$ which is defined by

$$
E_{\rho}(t):=\frac{\rho}{2}\left\|\partial_{t} u\right\|_{L^{2}}^{2}+\frac{\kappa}{2}\left\|\partial_{x}^{2} u\right\|_{L^{2}}^{2}+\frac{\alpha}{2}\left\|\partial_{x} u\right\|_{L^{2}}^{2}+\frac{\beta}{4}\left\|\partial_{x} u\right\|_{L^{2}}^{4}
$$

can be proved by the same arguments.

Lemma 3.1 (Decay of energy). Let $\eta \geq 0$. Assume that $(f, g) \in H^{2} \times L^{2}$. Then the solution to (1.2) constructed in Proposition 2.4 satisfies $E_{\rho}(t) \leq C_{2}(t+1)^{-1}$, where $C_{2}=$ $C\left(\|f\|_{H^{2}},\|g\|_{L^{2}}\right)$.

Proof. Multiplying (1.2) by $\partial_{t} u$ yields

$$
\partial_{t} E_{\rho}(t)+A(t)=0
$$

where $A(t):=\delta\left\|\partial_{t} u\right\|_{L^{2}}^{2}+\eta\left\|\partial_{t} \partial_{x}^{2} u\right\|_{L^{2}}^{2}+\gamma \eta\left(\int_{\mathbb{R}} \partial_{x} u \partial_{t} \partial_{x} u d x\right)^{2}$. Next, multiplying (1.2) by $u$, we obtain

$$
\begin{aligned}
\partial_{t} & \left(\frac{\eta}{2}\left\|\partial_{x}^{2} u\right\|_{L^{2}}^{2}+\rho \int_{\mathbb{R}} u \partial_{t} u d x+\frac{\gamma \eta}{4}\left\|\partial_{x} u\right\|_{L^{2}}^{4}+\frac{\delta}{2}\|u\|_{L^{2}}\right) \\
& +\kappa\left\|\partial_{x}^{2} u\right\|_{L^{2}}^{2}+\alpha\left\|\partial_{x} u\right\|_{L^{2}}^{2}+\beta\left\|\partial_{x} u\right\|_{L^{2}}^{4}=\rho\left\|\partial_{t} u\right\|_{L^{2}}^{2}
\end{aligned}
$$

Integrating the resulting equality (3.3) with respect to time variable over $[0, t]$, we have the following inequality

$$
\begin{aligned}
& \frac{\eta}{2}\left\|\partial_{x}^{2} u(t)\right\|_{L^{2}}^{2}+\frac{\gamma \eta}{4}\left\|\partial_{x} u(t)\right\|_{L^{2}}^{4}+\frac{\delta}{2}\|u(t)\|_{L^{2}}^{2} \leq \frac{\eta}{2}\left\|\partial_{x}^{2} f\right\|_{L^{2}}^{2}+\frac{\gamma \eta}{4}\left\|\partial_{x} f\right\|_{L^{2}}^{4}+\frac{\delta}{2}\|f\|_{L^{2}}^{2} \\
& +\rho\|f\|_{L^{2}}\|g\|_{L^{2}}+\rho\|u(t)\|_{L^{2}}\left\|\partial_{t} u(t)\right\|_{L^{2}}+\rho \int_{0}^{t}\left\|\partial_{t} u(s)\right\|_{L^{2}}^{2} d s \leq C+C\|u(t)\|_{L^{2}},
\end{aligned}
$$

with the help of the boundedness of $\left\|\partial_{t} u(t)\right\|_{L^{2}}$ and $\int_{0}^{t}\left\|\partial_{t} u(s)\right\|_{L^{2}}^{2} d s$, due to (3.2). Therefore we obtain

$$
\frac{\eta}{2}\left\|\partial_{x}^{2} u(t)\right\|_{L^{2}}^{2}+\frac{\gamma \eta}{4}\left\|\partial_{x} u(t)\right\|_{L^{2}}^{4}+\frac{\delta}{2}\|u(t)\|_{L^{2}}^{2} \leq C .
$$

We shall show that

$$
\sup _{s \in[t, t+1]} E_{\rho}(s) \leq C\left\{E_{\rho}(t)-E_{\rho}(t+1)\right\}+C \sqrt{E_{\rho}(t)-E_{\rho}(t+1)} .
$$

From (3.2) we have $E_{\rho}(t)-E_{\rho}(t+1)=\int_{t}^{t+1} A(s) d s$. Using the mean value theorem, there exist $\tau_{1} \in[t, t+1 / 4]$ and $\tau_{2} \in[t+3 / 4, t+1]$ satisfying $\frac{1}{4} A\left(\tau_{1}\right)=\int_{t}^{t+\frac{1}{4}} A(s) d s$ and $\frac{1}{4} A\left(\tau_{2}\right)=$ $\int_{t+\frac{3}{4}}^{t+1} A(s) d s$. Then we obtain

$$
\frac{1}{4} \max \left\{A\left(\tau_{1}\right), A\left(\tau_{2}\right)\right\} \leq E_{\rho}(t)-E_{\rho}(t+1)
$$


Here, from (3.3) we have

$$
\begin{aligned}
& \int_{\tau_{1}}^{\tau_{2}}\left(\rho\left\|\partial_{t} u\right\|_{L^{2}}^{2}+\kappa\left\|\partial_{x}^{2} u\right\|_{L^{2}}^{2}+\alpha\left\|\partial_{x} u\right\|_{L^{2}}^{2}+\beta\left\|\partial_{x} u\right\|_{L^{2}}^{4}\right) d s \\
& =-\delta \int_{\tau_{1}}^{\tau_{2}} \int_{\mathbb{R}}^{\tau_{2}} u \partial_{t} u d x d s+\rho \int_{\mathbb{R}} u\left(\tau_{1}\right) \partial_{t} u\left(\tau_{1}\right) d x-\rho \int_{\mathbb{R}} u\left(\tau_{2}\right) \partial_{t} u\left(\tau_{2}\right) d x \\
& \quad-\eta \int_{\tau_{1}}^{\tau_{\mathbb{R}}} \int_{\mathbb{R}}^{2} u \partial_{t} \partial_{x}^{2} u d x d s-\gamma \eta \int_{\tau_{1}}^{\tau_{2}}\left(\int_{\mathbb{R}} \partial_{x} u \partial_{t} \partial_{x} u d x \int_{\mathbb{R}}\left(\partial_{x} u\right)^{2} d x\right) d s+2 \rho \int_{\tau_{1}}^{\tau_{2}}\left\|\partial_{t} u(s)\right\|_{L^{2}}^{2} d s \\
& \leq \delta \int_{t}^{t+1}\|u(s)\|_{L^{2}}\left\|\partial_{t} u(s)\right\|_{L^{2}} d s+\rho\left\|u\left(\tau_{1}\right)\right\|_{L^{2}}\left\|\partial_{t} u\left(\tau_{1}\right)\right\|_{L^{2}}+\rho\left\|u\left(\tau_{2}\right)\right\|_{L^{2}}\left\|\partial_{t} u\left(\tau_{2}\right)\right\|_{L^{2}} \\
& \quad+\eta \int_{t}^{t+1}\left\|\partial_{x}^{2} u(s)\right\|_{L^{2}}\left\|\partial_{t} \partial_{x}^{2} u(s)\right\|_{L^{2}} d s+\gamma \eta \int_{t}^{t+1}\left|\int_{\mathbb{R}} \partial_{x} u \partial_{t} \partial_{x} u d x\right|\left\|\partial_{x} u(s)\right\|_{L^{2}}^{2} d s \\
& \quad+2 \rho \int_{t}^{t+1}\left\|\partial_{t} u(s)\right\|_{L^{2}}^{2} d s \\
& \leq C\left(\int_{t}^{t+1}\left\|\partial_{t} u(s)\right\|_{L^{2}}^{2} d s\right)^{\frac{1}{2}}+C\left\|\partial_{t} u\left(\tau_{1}\right)\right\|_{L^{2}}+C\left\|\partial_{t} u\left(\tau_{2}\right)\right\|_{L^{2}}+C\left(\int_{t}^{t+1}\left\|\partial_{t} \partial_{x}^{2} u(s)\right\|_{L^{2}}^{2} d s\right)^{\frac{1}{2}} \\
& \quad+C\left(\int_{t}^{t+1}\left|\int_{\mathbb{R}} \partial_{x} u \partial_{t} \partial_{x} u d x\right|^{\frac{1}{2}} d s\right)^{t+1}+2 \rho \int_{t}^{t}\left\|\partial_{t} u(s)\right\|_{L^{2}}^{2} d s \\
& \leq C \sqrt{E_{\rho}(t)-E_{\rho}(t+1)+C\left\{E_{\rho}(t)-E_{\rho}(t+1)\right\},}
\end{aligned}
$$

due to (3.4). Thus we conclude that

$$
\int_{\tau_{1}}^{\tau_{2}} E_{\rho}(s) d s \leq C \sqrt{E_{\rho}(t)-E_{\rho}(t+1)}+C\left\{E_{\rho}(t)-E_{\rho}(t+1)\right\} .
$$

By using the mean value theorem again, there exists $\tau_{0} \in\left[\tau_{1}, \tau_{2}\right]$ satisfying $\left(\tau_{2}-\tau_{1}\right) E_{\rho}\left(\tau_{0}\right)=$ $\int_{\tau_{1}}^{\tau_{2}} E_{\rho}(s) d s$. Since $\tau_{2}-\tau_{1} \geq 1 / 2>0$, we have $E_{\rho}\left(\tau_{0}\right) \leq C \sqrt{E_{\rho}(t)-E_{\rho}(t+1)}+C\left\{E_{\rho}(t)-\right.$ $\left.E_{\rho}(t+1)\right\}$. From (3.2) for any $\tau \in\left[\tau_{0}, t+1\right]$

$$
E_{\rho}\left(\tau_{0}\right)=E_{\rho}(\tau)+\int_{\tau_{0}}^{\tau} A(s) d s \geq E_{\rho}(\tau)
$$

and for any $\tau \in\left[t, \tau_{0}\right]$

$$
E_{\rho}(\tau) \leq E_{\rho}\left(\tau_{0}\right)+\int_{t}^{t+1} A(s) d s=E_{\rho}\left(\tau_{0}\right)+\left\{E_{\rho}(t)-E_{\rho}(t+1)\right\} .
$$

Combining (3.7) and (3.8) yields (3.5). It follows from (3.5) that

$$
\begin{aligned}
\sup _{s \in[t, t+1]} E_{\rho}(s)^{2} & \leq\left[C\left\{E_{\rho}(t)-E_{\rho}(t+1)\right\}+C \sqrt{E_{\rho}(t)-E_{\rho}(t+1)}\right]^{2} \\
& \leq C\left\{E_{\rho}(t)-E_{\rho}(t+1)\right\}^{2}+C\left\{E_{\rho}(t)-E_{\rho}(t+1)\right\} \\
& \leq C\left\{E_{\rho}(t)-E_{\rho}(t+1)\right\}\left\{E_{\rho}(t)-E_{\rho}(t+1)+1\right\} \\
& \leq C\left\{E_{\rho}(t)-E_{\rho}(t+1)\right\}\left\{E_{\rho}(t)+1\right\} .
\end{aligned}
$$

Then by Lemma 2.1 we have the desired result. 
From Lemma 3.1 we have the following a priori estimates:

$$
\|u(t)\|_{L^{2}} \leq C_{2}, \quad\left\|\partial_{x} u(t)\right\|_{L^{2}}+\left\|\partial_{t} u(t)\right\|_{L^{2}}+\left\|\partial_{x}^{2} u(t)\right\|_{L^{2}} \leq C_{2}(t+1)^{-\frac{1}{2}}
$$

By applying Proposition 2.3 to the Duhamel formula (2.2), we can increase the decay rate of $\left\|\partial_{t} u(t)\right\|_{L^{2}}$ and $\left\|\partial_{t} u(t)\right\|_{L^{2}}$, and particularly in the case $\eta>0$ also $\left\|\partial_{t} \partial_{x}^{2} u(t)\right\|_{L^{2}}$.

Lemma 3.2. Let $\eta \geq 0$. Assume that $(f, g) \in H^{2} \times L^{2}$. Then the solution for (1.2) satisfies

$$
\left\|\partial_{t} u(t)\right\|_{L^{2}}+\left\|\partial_{x}^{2} u(t)\right\|_{L^{2}} \leq \frac{C_{2}}{t+1}
$$

In addition, in the case $\eta>0$, if we assume $g \in H^{2}$, the solution also satisfies

$$
\left\|\partial_{x}^{2} \partial_{t} u(t)\right\| \leq \frac{\widetilde{C}_{2}}{(t+1)^{2}} .
$$

Proof. From (3.10) we see that $|I(u(t))|+|\widetilde{I}(u(t))| \leq C /(t+1)$. By applying Proposition 2.3 to the Duhamel formula (2.2), we have

$$
\begin{aligned}
\left\|\partial_{x}^{2} u(t)\right\| & \leq\left\|\partial_{x}^{2} \mathcal{K}_{1}(t) g\right\|_{L^{2}}+\left\|\partial_{x}^{2} \mathcal{K}_{0}(t) f\right\|_{L^{2}}+\int_{0}^{t} \frac{C}{s+1}\left\|\partial_{x}^{2} \mathcal{K}_{1}(t-s) \partial_{x}^{2} u(s)\right\|_{L^{2}} d s \\
& \leq \frac{C}{t+1}\left(\|f\|_{H^{2}}+\|g\|_{L^{2}}\right)+\int_{0}^{t} \frac{C\left\|\partial_{x} u(s)\right\|_{L^{2}}}{(s+1)(t-s+1)^{\frac{3}{2}}} d s+\int_{0}^{t} \frac{C\left\|\partial_{x}^{2} u(s)\right\|_{L^{2}}}{(s+1) e^{C(t-s)}} d s \\
& \leq \frac{C}{t+1}+\int_{0}^{t} \frac{C}{(t-s+1)^{\frac{3}{2}}(s+1)^{\frac{3}{2}}} d s+\int_{0}^{t} \frac{C}{(s+1)^{\frac{3}{2}} e^{C(t-s)}} d s \\
& \leq \frac{C}{t+1}+\frac{C}{(t+1)^{\frac{3}{2}}} \leq \frac{C}{t+1},
\end{aligned}
$$

thanks to Lemma 2.2, where we have used (2.7) of (2.11) as $k=4, \ell=3$ and $n=2$ in the nonlinear term. By differentiating (2.2) with respect to the time variable, we also obtain

$$
\begin{aligned}
\left\|\partial_{t} u(t)\right\| & \leq\left\|\partial_{t} \mathcal{K}_{1}(t) g\right\|_{L^{2}}+\left\|\partial_{t} \mathcal{K}_{0}(t) f\right\|_{L^{2}}+\int_{0}^{t} \frac{C}{s+1}\left\|\partial_{t} \mathcal{K}_{1}(t-s) \partial_{x}^{2} u(s)\right\|_{L^{2}} d s \\
& \leq \frac{C}{t+1}\left(\|f\|_{H^{2}}+\|g\|_{L^{2}}\right)+\int_{0}^{t} \frac{C\left\|\partial_{x} u(s)\right\|_{L^{2}}}{(s+1)(t-s+1)^{\frac{3}{2}}} d s+\int_{0}^{t} \frac{C\left\|\partial_{x}^{2} u(s)\right\|_{L^{2}}}{(s+1) e^{C(t-s)}} d s \\
& \leq \frac{C}{t+1}+\int_{0}^{t} \frac{C}{(t-s+1)^{\frac{3}{2}}(s+1)^{\frac{3}{2}}} d s+\int_{0}^{t} \frac{C}{(s+1)^{2} e^{C(t-s)}} d s \\
& \leq \frac{C}{t+1}+\frac{C}{(t+1)^{\frac{3}{2}}} \leq \frac{C}{t+1},
\end{aligned}
$$

thanks to $(2.8),(2.9)$ (or $(2.12),(2.13)$ ) and (3.13). Observe that the estimate (2.13) is the same as (2.9) if we choose $n=m=0$. This implies the estimate (3.11). 
Next, we show the decay estimate (3.12) for $\left\|\partial_{x}^{2} \partial_{t} u\right\|_{L^{2}}$ in the case $\eta>0$. By the Duhamel formula we obtain

$$
\begin{aligned}
& \left\|\partial_{x}^{2} \partial_{t} u(t)\right\|_{L^{2}} \leq \frac{C}{(t+1)^{2}}\left(\|f\|_{H^{2}}+\|g\|_{H^{2}}\right) \\
& \quad+\int_{0}^{t} \frac{C}{s+1}\left(\frac{\left\|\partial_{x}^{2} u(s)\right\|_{L^{2}}}{(t-s+1)^{\frac{2}{2}+1}}+\frac{\left\|\partial_{x}^{2} u(s)\right\|_{L^{2}}}{e^{C(t-s)}}+\frac{\left\|\partial_{x}^{2} u(s)\right\|_{L^{2}}}{(t-s)^{1 / 2} e^{C(t-s)}}\right) d s \\
& \leq \frac{C}{(t+1)^{2}}+\int_{0}^{t}\left(\frac{C}{(s+1)^{2}(t-s+1)^{2}}+\frac{C}{(s+1)^{2}(t-s)^{1 / 2} e^{C(t-s)}}\right) d s \leq \frac{C}{(t+1)^{2}},
\end{aligned}
$$

with the help of (2.13) in $\ell=m=n=2$. This completes the proof.

Higher-order energy estimates help us to show decay estimate of higher-order norms of the solution.

Lemma 3.3. Suppose that $k \geq 3$, and the exponents $\theta_{k}$ and $\widetilde{\theta}_{k}$ are given by (1.3).

1. Let $\eta=0$. If we assume $(f, g) \in H^{k} \times H^{k-2}$, the mild solution for (1.2) satisfies

$$
\left\|\partial_{x}^{k-2} \partial_{t} u(t)\right\|_{L^{2}}+\left\|\partial_{x}^{k} u(t)\right\|_{L^{2}} \leq \frac{C_{k}}{(t+1)^{\theta_{k}}} .
$$

2. Let $\eta>0$. If we assume $(f, g) \in H^{k} \times H^{k}$, the mild solution $u \in C\left([0, \infty) ; H^{k}\right) \cap$ $C^{1}\left([0, \infty) ; H^{k}\right)$ for $(1.2)$ satisfies

$$
\left\|\partial_{x}^{k} u(t)\right\|_{L^{2}} \leq \frac{\widetilde{C}_{k}}{(t+1)^{\widetilde{\theta}_{k}}}, \quad\left\|\partial_{x}^{k} \partial_{t} u(t)\right\|_{L^{2}} \leq \frac{\widetilde{C}_{k}}{(t+1)^{\widetilde{\theta}_{k+2}}} .
$$

Proof. The case $\eta>0$ is rather easy, we show it first. Let $k \geq 3$. From the Duhamel formula (2.2) and Proposition 2.3 with $n=3$ we see that

$$
\begin{gathered}
\left\|\partial_{x}^{k} u(t)\right\|_{L^{2}} \leq\left\|\partial_{x}^{k} \mathcal{K}_{1}(t) g\right\|_{L^{2}}+\left\|\partial_{x}^{k} \mathcal{K}_{0}(t) f\right\|_{L^{2}}+\int_{0}^{t} \frac{C}{s+1}\left\|\partial_{x}^{k} \mathcal{K}_{1}(t-s) \partial_{x}^{2} u(s)\right\|_{L^{2}} d s \\
\leq \frac{C}{(t+1)^{\frac{k}{2}}}\left(\|f\|_{H^{k}}+\|g\|_{H^{k}}\right)+\int_{0}^{t} \frac{C}{s+1}\left(\frac{\left\|\partial_{x}^{k+2-\ell} u(s)\right\|_{L^{2}}}{(t-s+1)^{\frac{\ell}{2}}}+\frac{\left\|\partial_{x}^{k-1} u(s)\right\|_{L^{2}}}{e^{C(t-s)}}\right) d s .
\end{gathered}
$$

For $k=3$, choosing $\ell=3$

$$
\int_{0}^{t} \frac{\left\|\partial_{x}^{2} u(s)\right\|_{L^{2}}}{(s+1)(t-s+1)^{\frac{3}{2}}} d s \leq \int_{0}^{t} \frac{C}{(s+1)^{2}(t-s+1)^{\frac{3}{2}}} d s \leq \frac{C}{(t+1)^{\frac{3}{2}}},
$$

so we obtain

$$
\left\|\partial_{x}^{3} u(t)\right\|_{L^{2}} \leq \frac{C}{(t+1)^{\frac{3}{2}}}+\frac{C}{(t+1)^{\frac{3}{2}}}+\frac{C}{(t+1)^{2}} \leq \frac{C}{(t+1)^{\frac{3}{2}}} .
$$

We show that $\widetilde{\theta}_{k}$ is non-decreasing. From the definition we immediately see that $\theta_{\widetilde{\sigma}}=2$. For some $k \geq 5$, we assume that $\widetilde{\theta}_{k-1} \geq \widetilde{\theta}_{k-2} \geq \cdots \geq \widetilde{\theta}_{0}$. Then from the definition of $\widetilde{\theta}_{k}$ we have

$$
\widetilde{\theta}_{k}=\max _{j=2, \ldots, k-1} \min \left\{\widetilde{\theta}_{(k-1)+2-j}+1, \frac{j+1}{2}\right\} \geq \max _{j=3, \ldots, k-1} \min \left\{\widetilde{\theta}_{(k-1)+2-j}+1, \frac{j}{2}\right\}=\widetilde{\theta}_{k-1},
$$


which implies $\widetilde{\theta}_{k} \geq \widetilde{\theta}_{k-1} \geq \cdots \geq \widetilde{\theta}_{0}$ for any $k$ by induction. Moreover, it follows from the definition and the non-decreasing property that

$$
\widetilde{\theta}_{k} \leq \min \left\{\widetilde{\theta}_{k-1}+1, k / 2\right\}
$$

Since from Lemma 2.2

$$
\int_{0}^{t} \frac{\left\|\partial_{x}^{k+2-\ell} u(s)\right\|_{L^{2}}}{(s+1)(t-s+1)^{\frac{\ell}{2}}} d s \leq \int_{0}^{t} \frac{C}{(s+1)^{\widetilde{\theta}_{k+2-\ell}+1}(t-s+1)^{\frac{\ell}{2}}} d s \leq \frac{C}{(t+1)^{\min \left\{\widetilde{\theta}_{k+2-\ell}+1, \ell / 2\right\}}}
$$

and

$$
\int_{0}^{t} \frac{\left\|\partial_{x}^{k-1} u(s)\right\|_{L^{2}}}{(s+1) e^{C(t-s)}} d s \leq \int_{0}^{t} \frac{C}{(s+1)^{\widetilde{\theta}_{k-1}+1} e^{C(t-s)}} d s \leq \frac{C}{(t+1)^{\widetilde{\theta}_{k-1}+1}}
$$

we obtain

$$
\left\|\partial_{x}^{k} u(t)\right\|_{L^{2}} \leq \frac{C}{(t+1)^{k / 2}}+\frac{C}{(t+1)^{\widetilde{\theta}_{k}}}+\frac{C}{(t+1)^{\tilde{\theta}_{k-1}+1}} \leq \frac{C}{(t+1)^{\widetilde{\theta}_{k}}},
$$

due to (3.14).

Similarly, from (2.12) and (2.13) with $n=m=2$,

$$
\begin{aligned}
& \left\|\partial_{x}^{k} \partial_{t} u(t)\right\|_{L^{2}} \leq\left\|\partial_{x}^{k} \partial_{t} \mathcal{K}_{1}(t) g\right\|_{L^{2}}+\left\|\partial_{x}^{k} \partial_{t} \mathcal{K}_{0}(t) f\right\|_{L^{2}}+\int_{0}^{t} \frac{C}{s+1}\left\|\partial_{x}^{k} \partial_{t} \mathcal{K}_{1}(t-s) \partial_{x}^{2} u(s)\right\|_{L^{2}} d s \\
& \leq \frac{C}{(t+1)^{\frac{k}{2}+1}}\left(\|f\|_{H^{k}}+\|g\|_{H^{k}}\right) \\
& \quad+\int_{0}^{t} \frac{C}{s+1}\left(\frac{\left\|\partial_{x}^{k+2-\ell} u(s)\right\|_{L^{2}}}{(t-s+1)^{\frac{\ell}{2}+1}}+\frac{\left\|\partial_{x}^{k} u(s)\right\|_{L^{2}}}{e^{C(t-s)}}+\frac{\left\|\partial_{x}^{k} u(s)\right\|_{L^{2}}}{(t-s)^{\frac{1}{2}} e^{C(t-s)}}\right) d s \\
& \leq \frac{C}{(t+1)^{\frac{k}{2}+1}}+\int_{0}^{t} \frac{C}{(s+1)^{\widetilde{\theta}_{k+2-\ell}+1}(t-s+1)^{\frac{\ell}{2}+1}} d s+\int_{0}^{t} \frac{C}{(s+1)^{\widetilde{\theta}_{k}+1} e^{C(t-s)}} d s \\
& \quad+\int_{0}^{t} \frac{C}{(s+1)^{\widetilde{\theta}_{k}+1}(t-s)^{\frac{1}{2}} e^{C(t-s)}} d s .
\end{aligned}
$$

We easily deduce that $\max _{\ell=3,4, \ldots, k+2} \min \left\{\widetilde{\theta}_{k+2-\ell}+1, \frac{\ell}{2}+1\right\}=\widetilde{\theta}_{k+2}$ and $\widetilde{\theta}_{k+2} \leq \widetilde{\theta}_{k}+1$. Therefore from Lemma 2.2 we conlude that

$$
\left\|\partial_{x}^{k} \partial_{t} u(t)\right\| \leq \frac{C}{(t+1)^{\frac{k+2}{2}}}+\frac{C}{(t+1)^{\widetilde{\theta}_{k+2}}}+\frac{C}{(t+1)^{\widetilde{\theta}_{k}+1}} \leq \frac{C}{(t+1)^{\widetilde{\theta}_{k+2}}},
$$

which completes the proof in the case $\eta>0$.

Next we show the estimate in the case $\eta=0$. By using the Duhamel formula (2.2), Lemma 2.2 and Proposition 2.3 again, we have

$$
\begin{gathered}
\left\|\partial_{x}^{k+2} u(t)\right\| \leq\left\|\partial_{x}^{k+2} \mathcal{K}_{1}(t) g\right\|_{L^{2}}+\left\|\partial_{x}^{k+2} \mathcal{K}_{0}(t) f\right\|_{L^{2}}+\int_{0}^{t} \frac{C}{s+1}\left\|\partial_{x}^{k+2} \mathcal{K}_{1}(t-s) \partial_{x}^{2} u(s)\right\|_{L^{2}} d s \\
\leq \frac{C}{(t+1)^{\frac{k+2}{2}}}\left(\|f\|_{H^{k+2}}+\|g\|_{H^{k}}\right)+\int_{0}^{t} \frac{C}{s+1}\left(\frac{\left\|\partial_{x}^{k+4-\ell} u(s)\right\|_{L^{2}}}{(t-s+1)^{\frac{\ell}{2}}}+\frac{\left\|\partial_{x}^{k+2} u(s)\right\|_{L^{2}}}{e^{C(t-s)}}\right) d s .
\end{gathered}
$$


Similarly, from (2.8) and (2.9)

$$
\begin{gathered}
\left\|\partial_{x}^{k} \partial_{t} u(t)\right\| \leq\left\|\partial_{x}^{k} \partial_{t} \mathcal{K}_{1}(t) g\right\|_{L^{2}}+\left\|\partial_{x}^{k} \partial_{t} \mathcal{K}_{0}(t) f\right\|_{L^{2}}+\int_{0}^{t} \frac{C}{s+1}\left\|\partial_{x}^{k} \partial_{t} \mathcal{K}_{1}(t-s) \partial_{x}^{2} u(s)\right\|_{L^{2}} d s \\
\leq \frac{C}{(t+1)^{\frac{k}{2}+1}}\left(\|f\|_{H^{k}}+\|g\|_{H^{k}}\right)+\int_{0}^{t} \frac{C}{s+1}\left(\frac{\left\|\partial_{x}^{k+2-\ell} u(s)\right\|_{L^{2}}}{(t-s+1)^{\frac{\ell}{2}+1}}+\frac{\left\|\partial_{x}^{k+2} u(s)\right\|_{L^{2}}}{e^{C(t-s)}}\right) d s .
\end{gathered}
$$

We write $u_{k}:=\partial_{x}^{k} u$ and

$$
E_{\rho, k}(t):=\frac{\rho}{2}\left\|\partial_{t} u_{k}\right\|_{L^{2}}^{2}+\frac{\kappa}{2}\left\|\partial_{x}^{2} u_{k}\right\|_{L^{2}}^{2}+\frac{\alpha}{2}\left\|\partial_{x} u_{k}\right\|_{L^{2}}^{2}+\frac{\beta}{2}\left\|\partial_{x} u\right\|_{L^{2}}^{2}\left\|\partial_{x} u_{k}\right\|_{L^{2}}^{4} .
$$

We shall show the decay of the energy (3.17) under the assumptions

$$
\left\|\partial_{x}^{k+1} u\right\|_{L^{2}} \leq \frac{C}{(t+1)^{\theta_{k-1}}}, \quad\left\|\partial_{x}^{k} u\right\|_{L^{2}} \leq \frac{C}{(t+1)^{\theta_{k-2}}} .
$$

By differentiating the equation (1.2), we see that

$$
\rho \partial_{t}^{2} u_{k}+\kappa \partial_{x}^{4} u_{k}+\delta \partial_{t} u_{k}=\left(\alpha+\beta \int_{\mathbb{R}}\left|\partial_{x} u\right|^{2} d x\right) \partial_{x}^{2} u_{k} .
$$

Multiplying with $\partial_{t} u_{k}$ yields

$$
\partial_{t} E_{\rho, k}(t)+\delta\left\|\partial_{t} u_{k}\right\|_{L^{2}}^{2}=B_{\rho, k}(t)
$$

where $B_{\rho, k}(t):=\beta\left\|\partial_{x} u_{k}\right\|_{L^{2}}^{2} \int_{\mathbb{R}} \partial_{t} \partial_{x} u \partial_{x} u d x$. Multiplying $u_{k}$ to (3.19) yields

$\delta \int_{\mathbb{R}} u_{k} \partial_{t} u_{k} d x+\rho \partial_{t} \int_{\mathbb{R}} u_{k} \partial_{t} u_{k} d x+\kappa\left\|\partial_{x}^{2} u_{k}\right\|_{L^{2}}^{2}+\alpha\left\|\partial_{x} u_{k}\right\|_{L^{2}}^{2}+\beta\left\|\partial_{x} u\right\|_{L^{2}}^{2}\left\|\partial_{x} u_{k}\right\|_{L^{2}}^{2}=\rho\left\|\partial_{t} u_{k}\right\|_{L^{2}}^{2}$

By the mean value theorem, there exist $\tau_{1} \in[t, t+1 / 4]$ and $\tau_{2} \in[t+3 / 4, t+1]$ satisfying

$$
\frac{1}{4}\left\|\partial_{t} u_{k}\left(\tau_{1}\right)\right\|_{L^{2}}^{2}=\int_{t}^{t+\frac{1}{4}}\left\|\partial_{t} u_{k}(s)\right\|_{L^{2}}^{2} d s, \quad \frac{1}{4}\left\|\partial_{t} u_{k}\left(\tau_{2}\right)\right\|_{L^{2}}^{2}=\int_{t+\frac{3}{4}}^{t+1}\left\|\partial_{t} u_{k}(s)\right\|_{L^{2}}^{2} d s .
$$

Then we obtain from (3.20)

$\frac{\delta}{4} \max \left\{\left\|\partial_{t} u_{k}\left(\tau_{1}\right)\right\|_{L^{2}}^{2},\left\|\partial_{t} u_{k}\left(\tau_{2}\right)\right\|_{L^{2}}^{2}\right\} \leq E_{\rho, k}(t)-E_{\rho, k}(t+1)+\int_{t}^{t+1} B_{\rho, k}(s) d s=: F_{\rho, k}(t)(\geq 0)$. 
Here from (3.21) we have

$$
\begin{gathered}
\int_{\tau_{1}}^{\tau_{2}}\left(\rho\left\|\partial_{t} u_{k}\right\|_{L^{2}}^{2}+\kappa\left\|\partial_{x}^{2} u_{k}\right\|_{L^{2}}^{2}+\alpha\left\|\partial_{x} u_{k}\right\|_{L^{2}}^{2}+\beta\left\|\partial_{x} u\right\|_{L^{2}}^{2}\left\|\partial_{x} u_{k}\right\|_{L^{2}}^{2}\right)(s) d s \\
\leq \delta \int_{t}^{t+1}\left\|u_{k}(s)\right\|_{L^{2}}\left\|\partial_{t} u_{k}(s)\right\|_{L^{2}} d s+\rho\left\|u_{k}\left(\tau_{1}\right)\right\|_{L^{2}}\left\|\partial_{t} u_{k}\left(\tau_{1}\right)\right\|_{L^{2}} \\
\quad+\rho\left\|u_{k}\left(\tau_{2}\right)\right\|_{L^{2}}\left\|\partial_{t} u_{k}\left(\tau_{2}\right)\right\|_{L^{2}}+2 \rho \int_{t}^{t+1}\left\|\partial_{t} u_{k}(s)\right\|_{L^{2}}^{2} d s \\
\leq \frac{C}{(t+1)^{\theta_{k-2}}}\left(\int_{t}^{t+1}\left\|\partial_{t} u_{k}(s)\right\|_{L^{2}}^{2} d s\right)^{\frac{1}{2}}+\frac{C}{(t+1)^{\theta_{k-2}}}\left\|\partial_{t} u_{k}\left(\tau_{1}\right)\right\|_{L^{2}} \\
\quad+\frac{C}{(t+1)^{\theta_{k-2}}}\left\|\partial_{t} u_{k}\left(\tau_{2}\right)\right\|_{L^{2}}+2 \rho \int_{t}^{t+1}\left\|\partial_{t} u_{k}(s)\right\|_{L^{2}}^{2} d s .
\end{gathered}
$$

Thus we conclude that $\int_{\tau_{1}}^{\tau_{2}} E_{\rho, k}(s) d s \leq \frac{C}{(t+1)^{\theta} k-2} \sqrt{F_{\rho, k}(t)}+C F_{\rho, k}(t)$. By using the mean value theorem, there exists $\tau_{0} \in\left[\tau_{1}, \tau_{2}\right]$ satisfying $\left(\tau_{2}-\tau_{1}\right) E_{\rho, k}\left(\tau_{0}\right)=\int_{\tau_{1}}^{\tau_{2}} E_{\rho, k}(s) d s$. Since $\tau_{2}-\tau_{1} \geq 1 / 2>0$, we see

$$
E_{\rho, k}\left(\tau_{0}\right) \leq \frac{C}{(t+1)^{\theta_{k-2}}} \sqrt{F_{\rho, k}(t)}+C F_{\rho, k}(t)
$$

It follows from integrating (3.20) that for any $\tau \in\left[\tau_{0}, t+1\right]$

$$
E_{\rho, k}\left(\tau_{0}\right)+\int_{\tau_{0}}^{\tau} B(s) d s=E_{\rho, k}(\tau)+\delta \int_{\tau_{0}}^{\tau}\left\|\partial_{t} u(s)\right\|_{L^{2}}^{2} d s \geq E_{\rho, k}(\tau) .
$$

Since $F_{\rho, k}(t)=\delta \int_{t}^{t+1}\left\|\partial_{t} u_{k}(s)\right\|_{L^{2}}^{2} d s$, we have for any $\tau \in\left[t, \tau_{0}\right]$

$$
E_{\rho, k}(\tau)=E_{\rho, k}\left(\tau_{0}\right)+\delta \int_{\tau}^{\tau_{0}}\left\|\partial_{t} u_{k}(s)\right\|_{L^{2}}^{2} d s-\int_{\tau}^{\tau_{0}} B_{\rho, k}(s) d s \leq E_{\rho, k}\left(\tau_{0}\right)+F_{\rho, k}(t)+\int_{t}^{t+1}\left|B_{\rho, k}(s)\right| d s .
$$

Combining (3.22) and (3.23) yields

$$
\sup _{\tau \in[t, t+1]} E_{\rho, k}(\tau) \leq \frac{C}{(t+1)^{\theta_{k-2}}} \sqrt{F_{\rho, k}(t)}+C F_{\rho, k}(t)+\int_{t}^{t+1}\left|B_{\rho, k}(s)\right| d s .
$$

From (3.11) and the assumption (3.18) we see that

$$
\left|B_{\rho, k}(t)\right| \leq\left\|\partial_{x} u_{k}(t)\right\|_{L^{2}}^{2}\left\|\partial_{t} u(t)\right\|_{L^{2}}\left\|\partial_{x}^{2} u(t)\right\|_{L^{2}} \leq \frac{C}{(t+1)^{2 \theta_{k-1}+2}},
$$

and from this we also have

$$
E_{\rho, k}(t+1)-E_{\rho, k}(t) \leq \frac{C}{(t+1)^{2 \theta_{k-1}+2}}, \quad E_{\rho, k}(t+1) \leq C .
$$


Therefore, we obtain

$$
\begin{aligned}
\sup _{\tau \in[t, t+1]} E_{\rho, k}(\tau)^{2} & \leq \frac{C}{(t+1)^{2 \theta_{k-2}}} F_{\rho, k}(t)+C F_{\rho, k}(t)^{2}+\frac{C}{(t+1)^{4 \theta_{k-1}+4}} \\
& \leq C\left(E_{\rho, k}(t)+\frac{1}{(t+1)^{2 \theta_{k-2}}}\right)\left\{E_{\rho, k}(t)-E_{\rho, k}(t+1)\right\}+\frac{C}{(t+1)^{2 \theta_{k-1}+2}},
\end{aligned}
$$

where we have used the fact that from (3.24)

$$
\begin{aligned}
& F_{\rho, k}(t)^{2} \leq C\left\{E_{\rho, k}(t)-E_{\rho, k}(t+1)\right\}^{2}+\frac{C}{(t+1)^{4 \theta_{k-1}+4}} \\
& =C E_{\rho, k}(t)\left\{E_{\rho, k}(t)-E_{\rho, k}(t+1)\right\}+C E_{\rho, k}(t+1)\left\{E_{\rho, k}(t+1)-E_{\rho, k}(t)\right\}+\frac{C}{(t+1)^{4 \theta_{k-1}+4}} \\
& \leq C E_{\rho, k}(t)\left\{E_{\rho, k}(t)-E_{\rho, k}(t+1)\right\}+\frac{C}{(t+1)^{2 \theta_{k-1}+2}}
\end{aligned}
$$

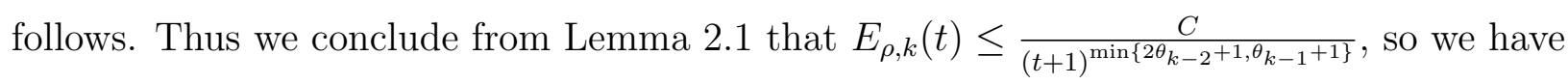

$$
\left\|\partial_{x}^{k} \partial_{t} u(t)\right\|_{L^{2}}+\left\|\partial_{x}^{k+2} u(t)\right\|_{L^{2}} \leq \frac{C}{(t+1)^{\min \left\{\theta_{k-2}+1 / 2,\left(\theta_{k-1}+1\right) / 2\right\}}} .
$$

For $k=1$, it holds that $\min \left\{\theta_{-1}+1 / 2,\left(\theta_{0}+1\right) / 2\right\}=1$. Substituting this into (3.15) with $k=1$ and $\ell=4$, we get

$$
\begin{aligned}
\left\|\partial_{x}^{3} u(t)\right\| & \leq \frac{C}{(t+1)^{\frac{3}{2}}}+\int_{0}^{t} \frac{C}{s+1}\left(\frac{\left\|\partial_{x} u(s)\right\|_{L^{2}}}{(t-s+1)^{2}}+\frac{\left\|\partial_{x}^{3} u(s)\right\|_{L^{2}}}{e^{C(t-s)}}\right) d s \\
& \leq \frac{C}{(t+1)^{\frac{3}{2}}}+\int_{0}^{t} \frac{C}{(t-s+1)^{2}(s+1)^{3 / 2}} d s+\int_{0}^{t} \frac{C}{e^{C(t-s)}(s+1)^{2}} d s \leq \frac{C}{(t+1)^{\frac{3}{2}}},
\end{aligned}
$$

and substituting it into (3.16) with $k=1$ and $\ell=2$, we have

$$
\left\|\partial_{x} \partial_{t} u(t)\right\| \leq \frac{C}{(t+1)^{\frac{3}{2}}}+\int_{0}^{t} \frac{C}{s+1}\left(\frac{\left\|\partial_{x} u(s)\right\|_{L^{2}}}{(t-s+1)^{2}}+\frac{\left\|\partial_{x}^{3} u(s)\right\|_{L^{2}}}{e^{C(t-s)}}\right) d s \leq \frac{C}{(t+1)^{\frac{3}{2}}} .
$$

So we have $\theta_{1}=3 / 2$. Similarly, for $k=2$, it holds that $\min \left\{\theta_{0}+1 / 2,\left(\theta_{1}+1\right) / 2\right\}=5 / 4$. Substituting this into (3.15) and (3.16) again, for $k=2, \ell=4$ and $k=2, \ell=2$, respectively, we have

$$
\begin{aligned}
\left\|\partial_{x}^{4} u(t)\right\| & \leq \frac{C}{(t+1)^{2}}+\int_{0}^{t} \frac{C}{s+1}\left(\frac{\left\|\partial_{x}^{2} u(s)\right\|_{L^{2}}}{(t-s+1)^{2}}+\frac{\left\|\partial_{x}^{4} u(s)\right\|_{L^{2}}}{e^{C(t-s)}}\right) d s \\
& \leq \frac{C}{(t+1)^{2}}+\int_{0}^{t} \frac{C}{(t-s+1)^{2}(s+1)^{2}} d s+\int_{0}^{t} \frac{C}{e^{C(t-s)}(s+1)^{1+\frac{5}{4}}} d s \leq \frac{C}{(t+1)^{2}},
\end{aligned}
$$

and

$$
\left\|\partial_{x}^{2} \partial_{t} u(t)\right\| \leq \frac{C}{(t+1)^{2}}+\int_{0}^{t} \frac{C}{s+1}\left(\frac{\left\|\partial_{x}^{2} u(s)\right\|_{L^{2}}}{(t-s+1)^{2}}+\frac{\left\|\partial_{x}^{4} u(s)\right\|_{L^{2}}}{e^{C(t-s)}}\right) d s \leq \frac{C}{(t+1)^{2}}
$$


So we have $\theta_{2}=2$. Similarly, in $k=3$, it holds that $\min \left\{\theta_{1}+1 / 2,\left(\theta_{2}+1\right) / 2\right\}=3 / 2$. Substituting this into (3.15) and (3.16) as $k=3, \ell=4$ and $k=3, \ell=2$, respectively, we have

$$
\begin{aligned}
&\left\|\partial_{x}^{5} u(t)\right\| \leq \frac{C}{(t+1)^{\frac{5}{2}}}+\int_{0}^{t} \frac{C}{s+1}\left(\frac{\left\|\partial_{x}^{3} u(s)\right\|_{L^{2}}}{(t-s+1)^{2}}+\frac{\left\|\partial_{x}^{5} u(s)\right\|_{L^{2}}}{e^{C(t-s)}}\right) d s \\
& \leq \frac{C}{(t+1)^{\frac{5}{2}}}+\int_{0}^{t} \frac{C}{(t-s+1)^{2}(s+1)^{\frac{5}{2}}} d s+\int_{0}^{t} \frac{C}{e^{C(t-s)}(s+1)^{1+\frac{5}{4}}} d s \leq \frac{C}{(t+1)^{2}}, \\
&\left\|\partial_{x}^{3} \partial_{t} u(t)\right\| \leq \frac{C}{(t+1)^{2}} .
\end{aligned}
$$

Then we see $\theta_{3}=2$. For $k \geq 4$, since $\theta_{k-2}=\theta_{k-1}=2$ which is the same situation as $k=3$, we only show by iterating the same procedure as above that $\left\|\partial_{x}^{k+2} u(t)\right\|_{L^{2}}+\left\|\partial_{x}^{k} \partial_{t} u(t)\right\|_{L^{2}} \leq$ $C(t+1)^{-2}(k \geq 4)$. This completes the proof of Lemma 3.3.

Acknowledgments. The second author is partially supported by the Japan Ministry of Education, Science, Sports and Culture, Grant-in-Aid for Scientific Research (C), No.25400172. The article was written when the second author visited the University of Konstanz between April 2014 and February 2015. He expresses his sincere gratitude to the kind hospitality of the people at the University of Konstanz.

\section{References}

[1] J. M. Ball, Stability theory for an extensible beam, J. Differential Equations, 14 (1973), 399-418.

[2] J. M. Ball, Initial-boundary value problems for an extensible beam, J. Math. Anal. Appl., 42 (1973), 61-90.

[3] P. Biler, Remark on the decay for damped string and beam equations, Nonlinear Anal. Theory, Methods and Appl., 10 (1986), 339-342.

[4] E.H. Brito, Decay estimates for the generalized damped extensible string and beam equations, Nonlinear Anal. Theory, Methods and Appl., 8 (1984), 1489-1496.

[5] R. W. Dickey, Free vibrations and dynamic buckling of the extensible beam, J. Math. Anal. Appl., 29 (1970), 443-454.

[6] J. G. Eisley, Nonlinear vibration of beams and rectangular plates, ZAMP, 15 (1964), 167-175.

[7] T. Hosono and T. Ogawa, Large time behavior and $L^{p}-L^{q}$ estimate of solutions of 2dimensional nonlinear damped wave equations, J. Differential Equations 203 (2004), 82-118. 
[8] S. Kawashima, M. Nakao and K. Ono, On the decay property of solutions to the Cauchy problem of the semilinear wave equation with a dissipative term, J. Math. Soc. Japan, 47 (1995), 617-653.

[9] M. Nakao, A difference inequality and its application to nonlinear evolution equations, J. Math. Soc. Japan, 30 (1978), 747-762.

[10] K. Ono, Asymptotic behavior of solutions for damped Kirchhoff equations in unbounded domain, Comm. Appl. Anal., 3 (1999), 101-114.

[11] H. Takeda and S. Yoshikawa, On the initial value problem of the semilinear beam equation with weak damping I: Smoothing effect, J. Math. Anal. Appl., 401 (2013) $244-258$.

[12] H. Takeda and S. Yoshikawa, On the initial value problem of the semilinear beam equation with weak damping Ii: Asymptotic profiles, J. Differential Equations, 253 (2012) 3061-3080.

[13] S. Woinovsky-Krieger, The effect of axial force on the vibration of hinged bars, J. Appl. Mech. 17 (1950), 35-36. 